\title{
Stationary Distribution and Extinction of a Stochastic Microbial Flocculation Model with Regime Switching
}

\author{
Haisu Zhang and Yi Song ii \\ College of Mathematics and Systems Science, Shandong University of Science and Technology, Qingdao 266590, China \\ Correspondence should be addressed to Yi Song; songyi_sy@sina.com
}

Received 9 August 2021; Accepted 2 November 2021; Published 22 November 2021

Academic Editor: Zhichao Jiang

Copyright (c) 2021 Haisu Zhang and Yi Song. This is an open access article distributed under the Creative Commons Attribution License, which permits unrestricted use, distribution, and reproduction in any medium, provided the original work is properly cited.

In this paper, a stochastic microbial flocculation model with regime switching is developed and analyzed. By proposing a suitable stochastic Lyapunov function, the existence and ergodicity of a stationary distribution for the system are proved. Then, the extinction of microorganisms is discussed under appropriate conditions and sufficient conditions for extinction are obtained. Finally, the results of the theoretical analysis are illustrated by numerical simulation.

\section{Introduction}

The flocculation process is a phenomenon in which small clumps usually aggregate into large clumps in a liquid medium [1]. Usually, a certain substance is added to the solid suspension, which can promote the occurrence of flocculation, and is generally called a flocculant. Flocculants have important applications in sewage treatment, energy extraction, biopharmacy, aquaculture, etc. [2, 3, 4]. For example, Gupta and Ako explored the effect of guar gum flocculant in the treatment of drinking water or food processing water through experiments and found that guar gum flocculant can not only improve the quality of water in the process of drinking water clarification, and there is no residue of acrylamide in the water, which reduces the health risks of the population [5]. Wu et al. used chitosan flocculant to treat the $\mathrm{Mn}$ (II) and suspended solids produced in the dual-alkali flue gas desulfurization regeneration process, which solved the problem that the traditional methods (such as chemical precipitation method, ion exchange method, adsorption method, and electrodeposition method) are difficult to remove heavy metal ions in the suspension [6]. Based on the flocculation precipitation method, Dharani and Balasubramanian synthesized a new water-soluble flocculant for harvesting microalgae, which is inexpensive, environmentally friendly, easy to synthesize, and has high harvesting efficiency for microalgae, compared with other flocculants [7].

The above research is generally based on experiments to study the flocculation effect of different flocculants or develop new flocculants. Recently, some scholars have begun to pay attention to the complex dynamic behavior in the process of microbial flocculation mathematically [8-17]. On the basis of the chemostat model [18-21], Tai et al. [9] proposed the following model:

$$
\left\{\begin{array}{l}
\frac{\mathrm{d} S(t)}{\mathrm{d} t}=D\left(S_{0}-S(t)\right)-\mu_{1} S(t) X(t) \\
\frac{\mathrm{d} X(t)}{\mathrm{d} t}=\mu_{2} S(t-\tau) X(t-\tau)-D X(t)-h_{2} X(t) P(t), \\
\frac{\mathrm{d} P(t)}{\mathrm{d} t}=D\left(P_{0}-P(t)\right)-h_{3} X(t) P(t),
\end{array}\right.
$$

to describe the process of microbial flocculation, where $S, X$, and $P$ represent the concentration of nutrition, microorganisms, and flocculants, respectively. For the meaning of all parameters, refer to Tai et al. [9]. Based on system (1) and motivated by the authors in [22, 23], Zhang et al. [14, 15] considered the following models from a random view: 


$$
\begin{aligned}
& \left\{\begin{array}{l}
\mathrm{d} S(t)=\left[D\left(S_{0}-S(t)\right)-\mu_{1} S(t) X(t)\right] \mathrm{d} t+\sigma_{1} S(t) \mathrm{d} B_{1}(t), \\
\mathrm{d} X(t)=\left[\mu_{2} S(t) X(t)-D X(t)-h_{2} X(t) P(t)\right] \mathrm{d} t+\sigma_{2} X(t) \mathrm{d} B_{2}(t), \\
\mathrm{d} P(t)=\left[D\left(P_{0}-P(t)\right)-h_{3} X(t) P(t)\right] \mathrm{d} t+\sigma_{3} P(t) \mathrm{d} B_{3}(t),
\end{array}\right. \\
& \left\{\begin{array}{l}
\mathrm{d} S(t)=\left[D\left(S_{0}-S(t)-\mu_{1} S(t) X(t)\right)\right] \mathrm{d} t+\sigma_{1} S(t) \mathrm{d} B_{1}(t), \\
\mathrm{d} X(t)=\left[\mu_{2} e^{-D \tau} S(t-\tau) X(t-\tau)-D X(t)-h_{2} X(t) P(t)\right] \mathrm{d} t+\sigma_{2} X(t) \mathrm{d} B_{2}(t), \\
\mathrm{d} P(t)=\left[D\left(P_{0}-P(t)\right)-h_{3} X(t) P(t)\right] \mathrm{d} t+\sigma_{3} P(t) \mathrm{d} B_{3}(t),
\end{array}\right.
\end{aligned}
$$

where $B_{i}(t)$ are independent standard Brownian motions and $B_{i}(0)=0 \quad(i=1,2,3)$ and $\sigma_{1}>0, \sigma_{2}>0$ and $\sigma_{3}>0$ are the intensities of the white noise on the nutrition, microorganisms, and flocculants, respectively. For system (2), the authors discussed the existence and ergodicity of a stationary distribution, and for system (3), the authors investigated the asymptotic behaviors of the solutions.

However, there is another kind of noise interference in nature, namely, telegraph noise or colored noise, which is used to express the transformation of system variables from one state to another. The development of microorganisms in a thermostat is not immune to the effects of temperature, humidity, or light. At the microscopic stage, the system constantly transitions from one state to another. Therefore, many researchers have focused on the chemostat model with telegraph noise and achieved good results [24-26].

In this paper, by using the method in Wang and Jiang [27], by considering the Markov regimes in the velocity $D$ in system (3), we get the new system as follows:

$$
\left\{\begin{array}{l}
\mathrm{d} S(t)=\left[D(r(t))\left(S_{0}-S(t)\right)-\mu_{1} S(t) X(t)\right] \mathrm{d} t+\sigma_{1}(r(t)) S(t) \mathrm{d} B_{1}(t) \\
\mathrm{d} X(t)=\left[\mu_{2} S(t) X(t)-D(r(t)) X(t)-h_{2} X(t) P(t)\right] \mathrm{d} t+\sigma_{2}(r(t)) X(t) \mathrm{d} B_{2}(t) \\
\mathrm{d} P(t)=\left[D(r(t))\left(P_{0}-P(t)\right)-h_{3} X(t) P(t)\right] \mathrm{d} t+\sigma_{3}(r(t)) P(t) \mathrm{d} B_{3}(t)
\end{array}\right.
$$

where $r(t), t \geq 0$ represent the continuous-time Markov chain independent from the Brownian motion $B(t)$ and takes a value in finite-state space $\mathbb{S}=\{1,2, \ldots, n\} . D(k)>0$ and $\sigma_{i}(k)>0, i=1,2,3$ hold for all $k \in \mathbb{S}$.

\section{Preliminary}

For a right-continuous Markov chain $r(t)$, the generator $\Upsilon=$ $\left(\gamma_{i j}\right)_{n \times n}$ is determined by

$$
P\{r(t+\Delta t)=j \mid r(t)=i\}= \begin{cases}\gamma_{i j} \Delta+o(\Delta), & \text { if } i \neq j, \\ 1+\gamma_{i j} \Delta+o(\Delta), & \text { if } i=j,\end{cases}
$$

where $\Delta t>0, \gamma_{i j} \geq 0$ is the transition rate from $i$ to $j$ if $i \neq j$ while $\gamma_{i i}=-\sum_{i \neq j} \gamma_{i j}$. In order to ensure that $r(t)$ is irreducible, here, we need to assume $\gamma_{i j} \geq 0$, for $i \neq j$. Thus, $r(t)$ has a unique stationary distribution $\pi=\left\{\pi_{1}, \pi_{2}, \ldots, \pi_{n}\right\}$ with the form

$$
\pi \Upsilon=0
$$

subject to

$$
\begin{aligned}
& \sum_{h=1}^{n} \pi_{h}=1 \\
& \pi_{h}>0, \quad \forall h \in \mathbb{S} .
\end{aligned}
$$

For a diffusion process $(u(t), r(t))$ expressed by stochastic differential equations,

$$
\left\{\begin{array}{l}
\mathrm{d} u(t)=g(u(t), r(t)) \mathrm{d} t+h(u(t), r(t)) \mathrm{d} B(t) \\
u(0)=u_{0}, r(0)=r
\end{array}\right.
$$

where $g(\cdot, \cdot): \mathbb{R}^{n} \times \mathbb{S} \longrightarrow \mathbb{R}^{n}, g(\cdot, \cdot): \mathbb{R}^{n} \times \mathbb{S} \longrightarrow \mathbb{R}^{n \times d}$ satisfying $h(u, k) h^{T}(u, k)=\left(p_{i j}(u, k)\right)$. For each $k \in \mathbb{S}$, let $V(\cdot, k)$ be any twice continuously differentiable function and we can define the operator $L$ as follows:

$$
L V(u, k)=\sum_{i=1}^{n} g_{i}(u, k) \frac{\partial V(u, k)}{\partial u_{i}}+\frac{1}{2} \sum_{i, j=1}^{n} p_{i j}(u, k) \frac{\partial^{2} V(u, k)}{\partial u_{i} \partial z_{j}}+\sum_{l=1}^{N} \gamma_{k l} V(u, l)
$$

To simplify, we define $\langle g(t)\rangle=(1 / t) \int_{0}^{t} g(s) \mathrm{d} s, t>0$, where $g(t)$ is an integral function define on $[0, \infty)$. And we define $g^{l}=\inf _{t \in[0, \infty)} g(t)$ and $g^{u}=\sup _{t \in[0, \infty)} g(t)$, where $g$ $(t)$ is a bounded function on $[0, \infty)$. We denote 
$\widehat{h}=\min _{k \in \mathbb{S}}\{h(k)\}$ and $\check{h}=\max _{k \in \mathbb{S}}\{h(k)\}$, where $\{h(k)\}_{k \in \mathbb{S}}$ is a constant vector. Then according to the ergodicity of Markov chain $r(\cdot)$, one gets

$$
\lim _{t \rightarrow \infty} \frac{1}{t} \int_{0}^{t} h(r(s)) \mathrm{d} s=\sum_{k \in \mathbb{S}} \pi_{k} h(k) \text { a.s. }
$$

\section{Existence and Uniqueness of Positive Solution}

Theorem 1. For any given initial value $(S(0), X(0), P(0), r(0)) \in \mathbb{R}_{+}^{3} \times \mathbb{S}$, there is a unique positive solution ( $S(t), X(t), P(t), r(t))$ of system (4) on $t \geq 0$ and the solution will remain in $\mathbb{R}_{+}^{3} \times \mathbb{S}$ with probability one.

Proof. Firstly, let $\hbar_{e}$ be the explosion time, we claim that there exists a unique local solution $(S(t), X(t), P(t), r(t)) \in \mathbb{R}_{+}^{3} \times \mathbb{S}$ with initial value $(S(0), X(0), P(0), r(0)) \in \mathbb{R}_{+}^{3} \times \mathbb{S}$ on $t \in\left[0, \hbar_{e}\right)$ a.s. In fact, it is easy to get from the local Lipschitz property of the coefficients of system (4).

Secondly, we prove $\hbar_{e}=\infty$ a.s.; it means the solution is global. Let $n_{0} \geq 1$ be sufficiently large such that $(S(0), X(0), P(0), r(0))$ all lie within the interval $\left[\left(1 / n_{0}\right), n_{0}\right]$ For each integer $n \geq n_{0}$, define the stopping time

$$
\hbar_{n}=\inf \left\{t \in\left[0, \hbar_{e}\right): S(t) \notin\left(\frac{1}{n}, n\right) \text { or } X(t) \notin\left(\frac{1}{n}, n\right) \text { or } P(t) \notin\left(\frac{1}{n}, n\right)\right\} \text {. }
$$

$$
\mathbb{P}\left\{\hbar_{\infty} \leq T\right\}>\epsilon .
$$

In the full text, we denote inf $\varnothing=\infty$ (the empty set). Clearly, $\hbar_{n}$ is increasing as $n \longrightarrow \infty$. Let $\hbar_{\infty}=\lim _{n \longrightarrow \infty} \hbar_{n}$, whence $\hbar_{\infty} \leq \hbar_{e}$ a.s. If $\hbar_{\infty}=\infty$ a.s. is true, then $\hbar_{e}=\infty$ and $(S(t), X(t), P(t), r(t)) \in \mathbb{R}_{+}^{3} \times \mathbb{S}$ a.s. for all $t \geq 0$. Then, to achieve our purpose, we only need to show $\hbar_{\infty}=\infty$ a.s. If this affirmation is not true, then there exist two constants Thus, for an integer $n_{1} \geq n_{0}$, we have

$$
\mathbb{P}\left\{\hbar_{n} \leq T\right\}>\epsilon, \quad \forall n \geq n_{1} .
$$

Define a $C^{2}$-function $V: \mathbb{R}_{+}^{3} \longrightarrow \mathbb{R}_{+}$as follows: $T>0$ and $\epsilon \in(0,1)$ such that

$$
V(S, X, P)=\left(S-a_{1}-a_{1} \ln \frac{S}{a_{1}}\right)+a_{2}\left(X-a_{3}-a_{3} \ln \frac{X}{a_{3}}\right)+P-a_{4}-a_{4} \ln \frac{P}{a_{4}}
$$

where $a_{1}=\left(2 \widehat{D} / 3 \mu_{2}\right), a_{2}=\left(\mu_{1} / \mu_{2}\right), a_{3}=\left(\mu_{2} \widehat{D} / \mu_{1} h_{2}\right), a_{4}=$ $\left(\mu_{1} \widehat{D} / 3 \mu_{2} h_{3}\right)$. From the Itô's formula, we have

$$
\begin{aligned}
\mathrm{d} V(S, X, P)= & \mathscr{L} V(S, X, P) \mathrm{d} t+\left(1-\frac{2 \widehat{D}}{3 \mu_{2} S}\right) \sigma_{1}(r(t)) S \mathrm{~d} B_{1}(t) \\
& +\left(\frac{\mu_{1}}{\mu_{2}}-\frac{\widehat{D}}{h_{2} X}\right) \sigma_{2}(r(t)) X \mathrm{~d} B_{2}(t) \\
& +\left(1-\frac{\mu_{1} \widehat{D}}{3 \mu_{2} h_{3} P}\right) \sigma_{3}(r(t)) P \mathrm{~d} B_{3}(t),
\end{aligned}
$$

where $\mathscr{L} V: \mathbb{R}_{+}^{3} \longrightarrow \mathbb{R}_{+}$is defined by 


$$
\begin{aligned}
\mathscr{L} V= & \left(1-\frac{2 \widehat{D}}{3 \mu_{2} S}\right)\left(D(k) S_{0}-D(k) S-\mu_{1} S X\right)+\left(\frac{\mu_{1}}{\mu_{2}}-\frac{\widehat{D}}{h_{2} X}\right)\left(\mu_{2} S X-D(k) X-h_{2} X P\right) \\
& +\left(1-\frac{\mu_{1} \widehat{D}}{3 \mu_{2} h_{3} P}\right)\left(D(k) P_{0}-D(k) P-h_{3} X P\right)+\frac{\widehat{D} \sigma_{1}^{2}(k)}{3 \mu_{2}}+\frac{\widehat{D} \sigma_{2}^{2}(k)}{2 h_{2}}+\frac{\mu_{1} \widehat{D} \sigma_{3}^{2}(k)}{6 \mu_{2} h_{3}} \\
= & D(k) S_{0}-D(k) S-\mu_{1} S X-\frac{2 \widehat{D} D(k) S_{0}}{3 \mu_{2} S}+\frac{2 \widehat{D} D(k)}{3 \mu_{2}}+\frac{2 \mu_{1} \widehat{D}}{3 \mu_{2}} X+\frac{\widehat{D} \sigma_{1}^{2}(k)}{3 \mu_{2}} \\
& +\mu_{1} S X-\frac{\mu_{1} D(k)}{\mu_{2}} X-\frac{\mu_{1} h_{2}}{\mu_{2}} X P-\frac{\mu_{2} \widehat{D}}{h_{2}} S+\frac{\widehat{D} D(k)}{h_{2}}+\widehat{D} P+\frac{\widehat{D} \sigma_{2}^{2}(k)}{2 h_{2}} \\
& +D(k) P_{0}-D(k) P-h_{3} X P-\frac{\mu_{1} \widehat{D} D(k) P_{0}}{3 \mu_{2} h_{3} P}+\frac{\mu_{1} \widehat{D} D(k)}{3 \mu_{2} h_{3}}+\frac{\mu_{1} \widehat{D}}{3 \mu_{2}} X+\frac{\mu_{1} \widehat{D} \sigma_{3}^{2}(k)}{6 \mu_{2} h_{3}} \\
\leq & \check{D} S_{0}+\frac{2 \widehat{D} \check{D}}{3 \mu_{2}}+\frac{\widehat{D} \check{D}}{h_{2}}+\check{D} P_{0}+\frac{\mu_{1} \widehat{D} \check{D}}{3 \mu_{2} h_{3}}+\frac{\widehat{D} \check{\sigma}_{1}^{2}}{3 \mu_{2}}+\frac{\widehat{D} \check{\sigma}_{2}^{2}}{2 h_{2}}+\frac{\mu_{1}}{6 \mu_{2} h_{3}^{2}}+\widehat{D} P-D(k) P+\frac{\mu_{1} \widehat{D}}{\mu_{2}} X-\frac{\mu_{1} D(k)}{\mu_{2}} X \\
:= & K_{1} \cdot \\
\leq & \check{D} S_{0}+\frac{2 \widehat{D} \check{D}}{3 \mu_{2}}+\frac{\widehat{D} \check{D}}{h_{2}}+\check{D} P_{0}+\frac{\mu_{1} \widehat{D} \check{D}}{3 \mu_{2} h_{3}}+\frac{\widehat{D} \check{\sigma}_{1}^{2}}{3 \mu_{2}}+\frac{\widehat{D} \check{\sigma}_{2}^{2}}{2 h_{2}}+\frac{\mu_{1} \widehat{D} \check{\sigma}_{3}^{2}}{6 \mu_{2} h_{3}} \\
&
\end{aligned}
$$

Integrating (15) from 0 to $T_{\min }=\hbar_{n} \wedge T=\min \left\{\hbar_{n}, T\right\}$ and taking the mathematical expectation on both sides, we have

$$
\mathbb{E} V\left(S\left(T_{\text {min }}\right), X\left(T_{\text {min }}\right), P\left(T_{\text {min }}\right)\right) \leq V(S(0), X(0), P(0))+K_{1} \mathbb{E}\left(T_{\text {min }}\right)
$$

Hence,

$$
\mathbb{E} V\left(S\left(T_{\min }\right), X\left(T_{\min }\right), P\left(T_{\min }\right)\right) \leq V(S(0), X(0), P(0))+K_{1} T
$$

Let $\Omega_{n}=\left\{\omega \in \Omega: \hbar_{n}=\hbar_{n}(\omega) \leq T\right\} \quad$ for $n \geq n_{1}$ and

$$
\frac{1}{n}-1-\ln \frac{1}{n}
$$
according to (13), we get $\mathbb{P}\left(\Omega_{n}\right) \geq \epsilon$. Note that, for every $\omega \in \Omega_{n}$, there exist $S\left(\hbar_{n}, \omega\right)$ or $X\left(\hbar_{n}, \omega\right)$ or $P\left(\hbar_{n}, \omega\right)$ equals either $1 / n$ or $n$. Therefore, $V\left(S\left(\hbar_{n}, \omega\right), X\left(\hbar_{n}, \omega\right), P\left(\hbar_{n}, \omega\right)\right)$ is no less than either

$$
\text { or } n-1-\ln n \text {. }
$$

Therefore, we have

$$
V\left(S\left(\hbar_{n}, \omega\right), X\left(\hbar_{n}, \omega\right), P\left(\hbar_{n}, \omega\right)\right) \geq\left(\frac{1}{n}-1+\ln n\right) \wedge(n-1-\ln n)
$$

By (18), we obtain

$$
V(S(0), X(0), P(0))+K_{1} T \geq \mathbb{E}\left[1_{\Omega_{n}(\omega)} V\left(S\left(\tau_{n}, \omega\right), X\left(\tau_{n}, \omega\right), P\left(\tau_{n}, \omega\right)\right)\right] \geq \varepsilon\left(\frac{1}{n}-1+\ln n\right) \wedge(n-1-\ln n)
$$


where $1_{\Omega_{n}}$ is the indicator function of $\Omega_{n}$. Let $n \longrightarrow \infty$, we obtain

$$
\infty>V(S(0), X(0), P(0))+K_{1} T=\infty,
$$

a contradiction. Thus, we obtain $\tau_{\infty}=\infty$ a.s. This completes the proof.

\section{Existence of an Ergodic Stationary Distribution}

In this section, we explore the existence and ergodicity of a stationary distribution for system (4). Firstly, by Lemma 3.2 in [28], we need to construct a new system, which has the same ergodic property and positive recurrence as the original system. To this end, we make a transformation $\alpha(t)=\log S(t), \beta(t)=\log X(t), \zeta(t)=\log P(t)$, then system (4) can be change to
Denote

$$
\Lambda(k)=\frac{D(k) S_{0} \mu_{2}}{D(k)+\left(\sigma_{1}^{2}(k) / 2\right)}-D(k)-h_{2} P_{0}-\frac{\sigma_{2}^{2}(k)}{2},
$$

and we have the following theorem.

Theorem 2. If $\Lambda=\sum_{k \in \mathbb{S}} \pi_{k} \Lambda(k)>0$, then the stochastic process $(S(t), X(t), P(t), r(t))$ of system (3) with any initial value $(S(0), X(0), P(0), r(0)) \in \mathbb{R}_{+}^{3} \times \mathbb{S}$ is ergodic and produce a unique stationary distribution in $\mathbb{R}_{+}^{3} \times \mathbb{S}$.

Proof. The proof is divided into three steps. We have to check whether the conditions of Lemma 2.1 in [28] are satisfied one by one. In the first step, by hypothesis $\gamma_{i j}>0$ for $i \neq j$, we can see condition (i) in Lemma 2.1 in [28] is met. Secondly, choose $g(z, k)=\operatorname{dig}\left(\sigma_{1}(k), \sigma_{2}(k), \sigma_{3}(k)\right)(k \in \mathbb{S})$, and then $g(z, k) g^{T}(z, k)=\operatorname{dig}\left(\sigma_{1}^{2}(k), \sigma_{2}^{2}(k), \sigma_{3}^{2}(k)\right)$ is positive definite; obviously, condition (ii) in Lemma 2.1 in [28] is met. At the last step, we will show condition (iii) in Lemma 2.1 in [28] is true. that

Let us choose a constant $\xi \in(0,1)$ sufficiently small such

$$
\text { (A) } \widehat{D}-\frac{\xi+1}{2}\left[\check{\sigma}_{1}^{2} \vee \check{\sigma}_{2}^{2} \vee \check{\sigma}_{3}^{2}\right]>0 \text {, }
$$

and $M>0$ sufficiently big such that

$$
\text { (B) } \Phi^{u}-M \Lambda \leq-2 \text {. }
$$

Now we construct a continuous function

where

$$
\tilde{V}(\alpha, \beta, \zeta, k)=M\left(V_{1}+V_{2}\right)+V_{3}+V_{4},
$$

$$
\begin{aligned}
& V_{1}=-a_{1} \alpha-\beta+\frac{h_{2}}{D(k)} e^{\zeta}+\frac{a_{1} \mu_{1}}{\widehat{D}} e^{\beta}, \\
& V_{2}=\varpi_{k}+|\Phi|, \\
& V_{3}=\frac{1}{\xi+2}\left(\mu_{2} e^{\alpha}+\mu_{1} e^{\beta}+e^{\zeta}\right)^{\xi+2}, \\
& V_{4}=-\zeta, \\
& a_{1}=\frac{D(k) S_{0} \mu_{2}}{\left(D(k)+\left(\sigma_{1}^{2}(k) / 2\right)\right)^{2}} .
\end{aligned}
$$

Since $\widetilde{V}(\widetilde{\alpha}, \widetilde{\beta}, \widetilde{\zeta}, k)$ is the minimum value point of function $\tilde{V}(\alpha, \beta, \zeta, k)$, then, $\widetilde{V}(\alpha, \beta, \zeta, k)-\widetilde{V}(\widetilde{\alpha}, \widetilde{\beta}, \widetilde{\zeta}, k) \geq 0$. We construct a $C^{2}$-function $V: \mathbb{R}_{+}^{3} \times \mathbb{S} \longrightarrow \mathbb{R}_{+}$with

$$
V(\alpha, \beta, \zeta, k)=\widetilde{V}(\alpha, \beta, \zeta, k)-\widetilde{V}(\widetilde{\alpha}, \widetilde{\beta}, \widetilde{\zeta}, k)+M\left(\Phi_{k}+|\Phi|\right),
$$

where $\omega=\left(\Phi_{1}, \Phi_{2}, \ldots,{\omega_{n}}^{T}, \quad|\bowtie|=\sqrt{\omega_{1}^{2}+\omega_{2}^{2}+\cdots+\omega_{n}^{2}}\right.$, and $\Phi_{k}(k \in \mathbb{S})$ will be determined as follows. $\omega$ here is used to make sure that $\Phi_{k}+\emptyset$ is nonnegative. Therefore, the $C^{2}$-function $V(\alpha, \beta, \zeta, k)$ is nonnegative.

By using the Itô's formula, we have

$$
L V(\alpha, \beta, \zeta, k)=M L\left(V_{1}+V_{2}\right)+L V_{3}+L V_{4} .
$$

From system (23) and by using Itô's formula, we have 


$$
\begin{aligned}
L( & \left.-a_{1} \alpha-\beta+\frac{h_{2}}{D(k)} e^{\zeta}\right) \\
= & -a_{1} D(k) S_{0} e^{-\alpha}+a_{1} D(k)+a_{1} \mu_{1} e^{\beta}+\frac{a_{1}}{2} \sigma_{1}^{2}(k)-\mu_{2} e^{\alpha}+D(k)+h_{2} e^{\zeta}+\frac{1}{2} \sigma_{2}^{2}(k) \\
& +h_{2} P_{0}-h_{2} e^{\zeta}-\frac{h_{2} h_{3} e^{\beta} e^{\zeta}}{D(k)} \\
\leq & -a_{1} D(k) S_{0} e^{-\alpha}-\mu_{2} e^{\alpha}+a_{1} \mu_{1} e^{\beta}+a_{1} D(k)+\frac{a_{1}}{2} \sigma_{1}^{2}(k)+h_{2} P_{0}+D(k)+\frac{1}{2} \sigma_{2}^{2}(k) \\
\leq & -2 \sqrt{a_{1} D(k) S_{0} \mu_{2}+a_{1}}\left(D(k)+\frac{\sigma_{1}^{2}(k)}{2}\right)+\left(D(k)+h_{2} P_{0}+\frac{\sigma_{2}^{2}(k)}{2}\right)+a_{1} \mu_{1} e^{\beta} \\
\leq & -\left(\frac{D(k) S_{0} \mu_{2}}{D(k)+\left(\sigma_{1}^{2}(k) / 2\right)}-D(k)-h_{2} P_{0}-\frac{\sigma_{2}^{2}(k)}{2}\right)+a_{1} \mu_{1} e^{\beta} \\
:= & -\Lambda(k)+a_{1} \mu_{1} e^{\beta} .
\end{aligned}
$$

In view of the second equation on (23), we have

$$
L e^{\beta}=\mu_{2} e^{\alpha} e^{\beta}-D(k) e^{\beta}-h_{2} e^{\zeta} e^{\beta} \leq \mu_{2} e^{\alpha} e^{\beta}-\widehat{D} e^{\beta} .
$$

Therefore, we get

$$
L V_{1} \leq-\Lambda(k)+\frac{a_{1} \mu_{1} \mu_{2}}{\widehat{D}} e^{\alpha} e^{\beta} .
$$

Similarly, according to the Itô's formula, we have

$$
L V_{2}=\sum_{l \neq k \in \mathbb{S}} \gamma_{k l}\left(\varpi_{l}-\varpi_{k}\right)
$$

Next, let us define the vector $\Omega=\left(\Omega_{1}, \Omega_{2}, \ldots, \Omega_{n}\right)$ with elements $\Omega_{k}=-\Lambda_{1}(k), k=1,2, \ldots, n$. Since the generator matrix $\Upsilon$ is irreducible, then for each $\Lambda_{k}$, there exists one solution $\omega=\left(\varpi_{1}, \varpi_{2}, \ldots, \varpi_{n}\right)^{T}$ of the Poisson system (see Lemma 2.3 [29]) such that

$$
\Upsilon ®+\Omega=\sum_{j=1}^{n} \pi_{j} \Omega_{j} \overrightarrow{1}
$$

where $\overrightarrow{1}$ is the unit vector. Taking advantage of $\pi \Upsilon=0$, one gets

$$
-\Lambda(k)+\sum_{l \neq k \in \mathbb{S}} \gamma_{k l}\left(\oplus_{l}-\varpi_{k}\right)=-\sum_{k \in \mathbb{S}} \pi_{k} \Lambda(k)=-\Lambda .
$$

Then, we obtain that

$$
L\left(V_{1}+V_{2}\right) \leq-\Lambda+\frac{a_{1} \mu_{1} \mu_{2}}{\widehat{D}} e^{\alpha} e^{\beta} .
$$

In the meanwhile, denote $Q(\alpha, \beta, \zeta)=\mu_{2} e^{\alpha}+\mu_{1} e^{\beta}+e^{\zeta}$, and we have

$$
\begin{aligned}
L V_{3} \leq & Q^{\xi+1}(\alpha, \beta, \zeta)\left[D(k) \mu_{2} S_{0}-D(k) \mu_{2} e^{\alpha}-D(k) \mu_{1} e^{\beta}+D(k) P_{0}-D(k) e^{\zeta}\right] \\
& +\frac{\xi+1}{2} \sigma_{1}^{2}(k)\left(\mu_{2} e^{\alpha}\right)^{2} Q^{\xi}(\alpha, \beta, \zeta)+\frac{\xi+1}{2} \sigma_{2}^{2}(k)\left(\mu_{1} e^{\beta}\right)^{2} Q^{\xi}(\alpha, \beta, \zeta)+\frac{\xi+1}{2} \sigma_{3}^{2}(k)\left(e^{\zeta}\right)^{2} Q^{\xi}(\alpha, \beta, \zeta) \\
\leq & \left(\check{D} \mu_{2} S_{0}+\check{D} P_{0}\right) Q^{\xi+1}(\alpha, \beta, \zeta)-\widehat{D} Q^{\xi+2}(\alpha, \beta, \zeta)+\frac{\xi+1}{2} Q^{\xi}(\alpha, \beta, \zeta)\left[\check{\sigma}_{1}^{2} \vee \check{\sigma}_{2}^{2} \vee \check{\sigma}_{3}^{2}\right] Q^{2}(\alpha, \beta, \zeta) \\
= & \left(\check{D} \mu_{2} S_{0}+\check{D} P_{0}\right) Q^{\xi+1}(\alpha, \beta, \zeta)-\left[\widehat{D}-\frac{\xi+1}{2}\left(\check{\sigma}_{1}^{2} \vee \check{\sigma}_{1}^{2} \vee \check{\sigma}_{3}^{2}\right)\right] Q^{\xi+2}(\alpha, \beta, \zeta) .
\end{aligned}
$$

By using the Itô's formula to $V_{4}$, we obtain 


$$
\begin{aligned}
L V_{4} & =-\frac{D(k) P_{0}}{e^{\zeta}}+D(k)+h_{3} e^{\beta}+\frac{1}{2} \sigma_{3}^{2}(k) \\
& \leq-\frac{\widehat{D} P_{0}}{e^{\zeta}}+\check{D}+h_{3} e^{\beta}+\frac{1}{2} \check{\sigma}_{3}^{2} .
\end{aligned}
$$

According (30) and (37)-(39), we have

$$
L V \leq \Phi(\alpha, \beta, \zeta)+\Psi(\alpha, \beta),
$$

where

$$
\begin{gathered}
\Phi(\alpha, \beta, \zeta)=\left(\check{D} \mu_{2} S_{0}+\check{D} P_{0}\right)\left(\mu_{2} e^{\alpha}+\mu_{1} e^{\beta}+e^{\zeta}\right)^{\xi+1}-\frac{\widehat{D} P_{0}}{e^{\zeta}}+\check{D}+h_{3} e^{\beta}+\frac{1}{2} \check{\sigma}_{3}^{2} \\
-\left[\widehat{D}-\frac{\xi+1}{2}\left(\check{\sigma}_{1}^{2} \vee \check{\sigma}_{1}^{2} \vee \check{\sigma}_{3}^{2}\right)\right]\left(\mu_{2} e^{\alpha}+\mu_{1} e^{\beta}+e^{\zeta}\right)^{\xi+2}, \\
\Psi(\alpha, \beta)=M\left(-\Lambda+\frac{a_{1} \mu_{1} \mu_{2}}{\widehat{D}} e^{\alpha} e^{\beta}\right) .
\end{gathered}
$$

According to condition $(A)$, we derive that

$$
\begin{aligned}
\lim _{\zeta \longrightarrow+\infty} \Phi(\alpha, \beta, \zeta)+\Psi^{u} & =-\infty, \lim _{\beta \longrightarrow+\infty} \Phi(\alpha, \beta, \zeta)+\lim _{\beta \longrightarrow+\infty} \Psi(\alpha, \beta) \\
& =-\infty, \lim _{\alpha \longrightarrow+\infty} \Phi(\alpha, \beta, \zeta)+\lim _{\alpha \longrightarrow+\infty} \Psi(\alpha, \beta) \\
& =-\infty, \lim _{\zeta \longrightarrow-\infty} \Phi(\alpha, \beta, \zeta)+\Psi^{u}
\end{aligned}
$$

In the same way, we get

$$
\begin{aligned}
& \lim _{\beta \longrightarrow-\infty} \Phi(\alpha, \beta, \zeta)+\lim _{\beta \longrightarrow-\infty} \Psi(\alpha, \beta)=\Phi^{u}-M \Lambda \leq-2, \\
& \alpha \longrightarrow-\infty
\end{aligned}
$$

Let us consider a compact subset $U \subset \mathbb{R}_{+}^{3}$ as follows:

$$
U=\left\{\log \epsilon<\alpha<\log \frac{1}{\epsilon}, \log \epsilon<\beta<\log \frac{1}{\epsilon}, \log \epsilon<\zeta<\log \frac{1}{\epsilon}\right\},
$$

where $\epsilon$ is small enough. Then we obtain

$$
L V(\alpha, \beta, \zeta, k)<-1,(\alpha, \beta, \zeta, k) \in\left\{\mathbb{R}_{+}^{3} \backslash U\right\} \times \mathbb{S} .
$$

In summary, the conditions of Lemma 2.1 in [28] are fully satisfied, and then we conclude that diffusion process $(\alpha(t), \beta(t), \zeta(t), r(t))$ for system (23) is ergodic. Hence, stochastic process $(S(t), X(t), P(t), r(t))$ produces an ergodic stationary distribution. The proof is completed.

\section{Extinction}

Lemma 1 (see [30]). Let $N=\left\{N_{t}\right\}_{t} \geq 0$ be a real-valued continuous local martingale vanishing at $t=0$. Then,

$$
\begin{array}{ll}
\lim _{t \longrightarrow \infty}\langle N, N\rangle_{t}=\infty & \text { a.s. } \Rightarrow \lim _{t \longrightarrow \infty} \frac{N_{t}}{\langle N, N\rangle_{t}}=0 \text { a.s., } \\
\lim _{t \longrightarrow \infty} \frac{\langle N, N\rangle_{t}}{t}<\infty & \text { a.s. } \Rightarrow \lim _{t \longrightarrow \infty} \frac{N_{t}}{t}=0 \text { a.s. }
\end{array}
$$

The following lemma is essentially the same as that in [31], so we omit it.

Lemma 2. Let $(S(t), X(t), P(t), r(t))$ be the solution of system (3) with any initial value $(S(0), X(0), P(0), r(0)) \in \mathbb{R}_{+}^{3} \times \mathbb{S}$ if the following hypothesis $\left(K_{1}\right)$ holds.

$\left(K_{1}\right)$ : there is a constant $\iota>2$ such that

$$
\widehat{D}-\frac{1}{2}(\iota-1)\left(\check{\sigma}_{1}^{2} \vee \check{\sigma}_{2}^{2} \vee \check{\sigma}_{3}^{2}\right)>0,
$$


then the solution $(S(t), X(t), P(t), r(t))$ has the following properties:

$$
\begin{aligned}
& \lim _{t \rightarrow \infty} \frac{S(t)}{t}=0, \\
& \lim _{t \rightarrow \infty} \frac{X(t)}{t}=0, \\
& \lim _{t \rightarrow \infty} \frac{P(t)}{t}=0,
\end{aligned}
$$

$$
\begin{aligned}
& \lim _{t \rightarrow \infty} \frac{1}{t} \int_{0}^{t} \sigma_{1}(r(s)) S(s) \mathrm{d} B_{1}(s)=0, \\
& \lim _{t \rightarrow \infty} \frac{1}{t} \int_{0}^{t} \sigma_{1}(r(s)) X(s) \mathrm{d} B_{2}(s)=0, \\
& \lim _{t \rightarrow \infty} \frac{1}{t} \int_{0}^{t} \sigma_{1}(r(s)) P(s) \mathrm{d} B_{3}(s)=0, \text { a.s. }
\end{aligned}
$$

Theorem 3. Let hypothesis $\left(K_{1}\right)$ hold. If $\mu_{2} S_{0}(\check{D} / \widehat{D})+P_{0}(\check{D} / \widehat{D})<\sum_{k \in \mathbb{S}} \pi_{k}\left[D(k)+(1 / 2) \sigma_{2}^{2}(k)\right]$, then the solution $(S(t), X(t), P(t), r(t))$ for stochastic system (4) with any initial value $(S(0), X(0), P(0), r(0)) \in \mathbb{R}_{+}^{3} \times \mathbb{S}$, will have the property that

$$
\lim _{t \rightarrow \infty} X(t)=0
$$

a.s., which implies the microorganism $X(t)$ tends to extinction exponentially with probability one.

Proof. Respectively, from each equation of system (4), we can obtain

$$
\begin{aligned}
\frac{\mu_{2}(S(t)-S(0))}{t} & =\mu_{2} S_{0}\langle D(r(t))\rangle-\mu_{2}\langle D(r(t)) S(t)\rangle-\mu_{1} \mu_{2}\langle S(t) X(t)\rangle+\frac{\mu_{2}}{t} \int_{0}^{t} \sigma_{1}(r(s)) S(s) \mathrm{d} B_{1}(s) \\
\frac{\mu_{1}(X(t)-X(0))}{t} & =\mu_{1} \mu_{2}\langle S(t) X(t)\rangle-\mu_{1}\langle D(r(t)) X(t)\rangle-\mu_{1} h_{2}\langle X(t) P(t)\rangle+\frac{\mu_{1}}{t} \int_{0}^{t} \sigma_{1}(r(s)) X(s) \mathrm{d} B_{2}(s) \\
\frac{P(t)-P(0)}{t} & =P_{0}\langle D(r(t))\rangle-\langle D(r(t)) P(t)\rangle-h_{3}\langle X(t) P(t)\rangle+\frac{1}{t} \int_{0}^{t} \sigma_{1}(r(s)) P(s) \mathrm{d} B_{3}(s) .
\end{aligned}
$$

Add the left side of the three equations above to get

$$
\begin{aligned}
& \frac{\mu_{2}(S(t)-S(0))}{t}+\frac{\mu_{1}(X(t)-X(0))}{t}+\frac{P(t)-P(0)}{t} \\
& \leq \mu_{2} S_{0}\langle D(r(t))\rangle-\mu_{2}\langle D(r(t)) S(t)\rangle-\mu_{1}\langle D(r(t)) X(t)\rangle+P_{0}\langle D(r(t))\rangle-\langle D(r(t)) P(t)\rangle+\omega_{1}+\omega_{2}+\omega_{3},
\end{aligned}
$$

where

$$
\begin{aligned}
& \omega_{1}=\frac{\mu_{2}}{t} \int_{0}^{t} \sigma_{1}(r(s)) S(s) \mathrm{d} B_{1}(s), \\
& \omega_{2}=\frac{\mu_{1}}{t} \int_{0}^{t} \sigma_{1}(r(s)) X(s) \mathrm{d} B_{2}(s), \\
& \omega_{3}=\frac{1}{t} \int_{0}^{t} \sigma_{1}(r(s)) P(s) \mathrm{d} B_{3}(s),
\end{aligned}
$$

$$
\mu_{2}\langle D(r(t)) S(t)\rangle+\mu_{1}\langle D(r(t)) X(t)\rangle+\langle D(r(t)) P(t)\rangle \leq \mu_{2} S_{0}\langle D(r(t))\rangle+P(0)\langle D(r(t))\rangle-\varphi_{1}(t),
$$

In the sequel, 


$$
\mu_{2}\langle S(t)\rangle+\mu_{1}\langle X(t)\rangle+\langle P(t)\rangle \leq \mu_{1} S_{0} \frac{\check{D}}{\widehat{D}}+P_{0} \frac{\check{D}}{\widehat{D}}-\frac{\varphi_{1}(t)}{\widehat{D}}, \quad \text { where }
$$

$$
\varphi_{1}(t)=\frac{\mu_{2}(S(t)-S(0))}{t}+\frac{\mu_{1}(X(t)-X(0))}{t}+\frac{P(t)-P(0)}{t}-\omega_{1}-\omega_{2}-\omega_{3} .
$$

According to Lemma 2, we have

$$
\lim _{t \rightarrow \infty} \varphi_{1}(t)=0 \text { a.s. }
$$

By using the Itô's formula and utilizing (54), we derive that

$$
\begin{aligned}
\ln X(t)= & \left.\ln X(0)+\mu_{2}\langle S(t)\rangle t-\langle D(r(t))\rangle+\frac{1}{2} \sigma_{2}^{2}(r(t))\right\rangle t-h_{2}\langle P(t)\rangle t+\int_{0}^{t} \sigma_{2}(r(s)) \mathrm{d} B_{2}(s) \\
\leq & \ln X(0)-\mu_{1}\langle X(t)\rangle t-\langle P(t)\rangle t+\mu_{1} S_{0} \frac{\check{D}}{\widehat{D}} t+P_{0} \frac{\check{D}}{\widehat{D}} t \\
& \quad-\frac{\varphi_{1}(t)}{\widehat{D}} t-\left\langle D(r(t))+\frac{1}{2} \sigma_{2}^{2}(r(t))\right\rangle t+\int_{0}^{t} \sigma_{2}(r(s)) \mathrm{d} B_{2}(s) \\
\leq & \left.\ln X(0)+\mu_{1} S_{0} \frac{\check{D}}{\widehat{D}} t+P_{0} \frac{\check{D}}{\widehat{D}} t-\frac{\varphi_{1}(t)}{\widehat{D}} t-\langle D(r(t))\rangle+\frac{1}{2} \sigma_{2}^{2}(r(t))\right\rangle t+\int_{0}^{t} \sigma_{2}(r(s)) \mathrm{d} B_{2}(s) .
\end{aligned}
$$

Let us divide by $t$ on both sides of the above formula, and we can get

$$
\frac{\ln X(t)}{t} \leq \frac{\ln X(0)}{t}+\mu_{1} S_{0} \frac{\check{D}}{\widehat{D}}+P_{0} \frac{\check{D}}{\widehat{D}}-\frac{\varphi_{1}(t)}{\widehat{D}}-\left\langle D(r(t))+\frac{1}{2} \sigma_{2}^{2}(r(t))\right\rangle+\frac{M_{2}(t)}{t}
$$

where $M_{i}(t)=\int_{0}^{t} \sigma_{i}(r(s)) \mathrm{d} B_{i}(s)(i=1,2,3)$ satisfies

$$
\lim _{t \rightarrow \infty} \frac{M_{i}(t)}{t}=0 \text { a.s. }
$$

$$
\left\langle M_{i}, M_{i}\right\rangle=\int_{0}^{t} \sigma_{i}^{2}(r(s)) \mathrm{d} s<\check{\sigma}_{i}^{2} t .
$$

On the other hand, from (10), we have

Together with Lemma 1, we can get

$$
\lim _{t \longrightarrow \infty} \frac{1}{t} \int_{0}^{t}\left[D(r(s))+\frac{1}{2} \sigma_{2}^{2}(r(s))\right] \mathrm{d} s=\sum_{k \in \mathbb{S}} \pi_{k}\left[D(k)+\frac{1}{2} \sigma_{2}^{2}(k)\right] .
$$

Take the limit on both sides of (58); if $\mu_{2} S_{0}(\check{D} / \widehat{D})+P_{0}(\check{D} / \widehat{D})<\sum_{k \in \mathbb{S}} \pi_{k}\left[D(k)+(1 / 2) \sigma_{2}^{2}(k)\right]$, then we have

$$
\limsup _{t \rightarrow \infty} \frac{\ln X(t)}{t} \leq \mu_{2} S_{0} \frac{\check{D}}{\widehat{D}}+P_{0} \frac{\check{D}}{\widehat{D}}-\sum_{k \in \mathbb{S}} \pi_{k}\left[D(k)+\frac{1}{2} \sigma_{2}^{2}(k)\right]<0 \text { a.s. }
$$




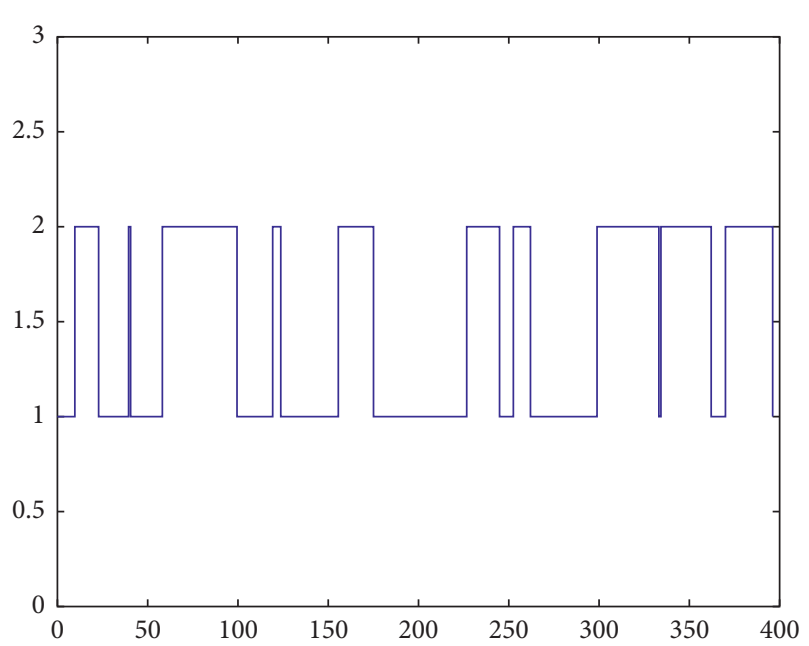

(a)

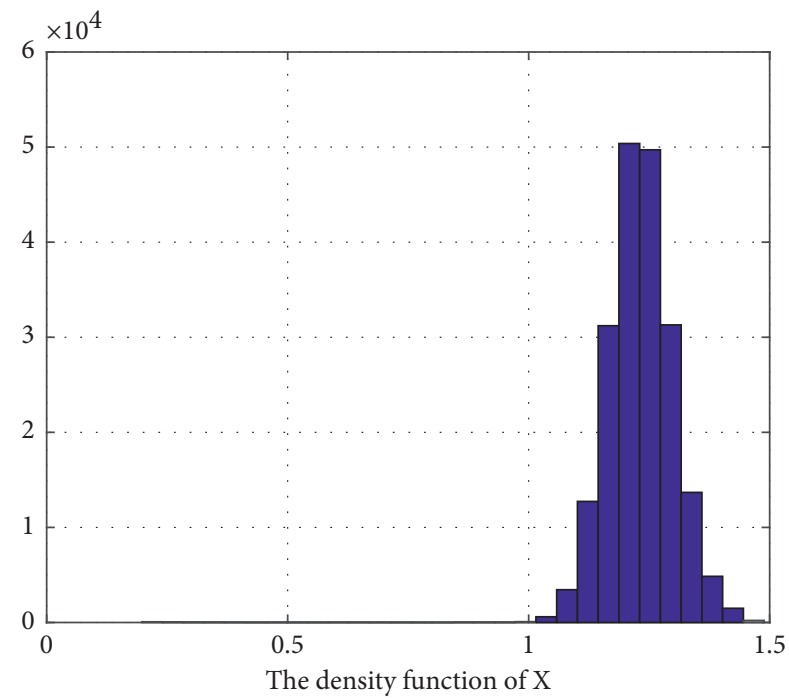

(c)

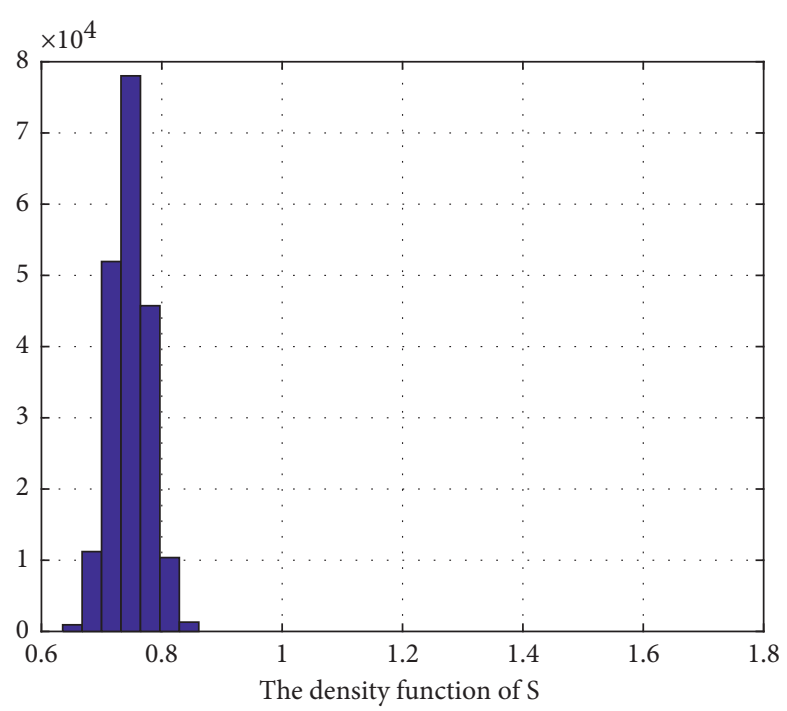

(b)

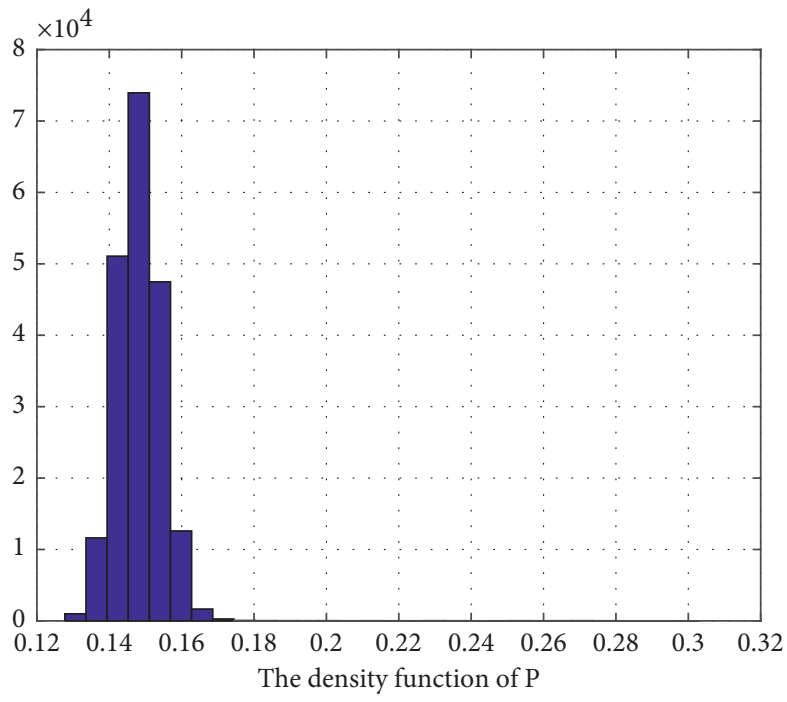

(d)

FIgURE 1: Numerical simulation of system (4). (a) Markov chain, (b) the density function of $S$, (c) the density function of $X$, and (d) the density function of $P$.

Hence, we get

$$
\lim _{t \rightarrow \infty} X(t)=0 \text { a.s. }
$$

This completes the proof.

\section{Numerical Simulations}

Now, we use numerical simulations to verify the results previously obtained. A discretization for system (3) implies

$$
\left\{\begin{array}{l}
S_{k+1}=S_{k}+\left[D(r(i \Delta t))\left(S_{0}-S_{k}\right)-\mu_{1} S_{k} X_{k}\right] \Delta t+\sigma_{1}(r(i \Delta t)) S_{k} \Delta B_{1, k}, \\
X_{k+1}=X_{k}+\left[\mu_{2} S_{k} X_{k}-D(r(i \Delta t)) X_{k}-h_{2} X_{k} P_{k}\right] \Delta t+\sigma_{2}(r(i \Delta t)) X_{k} \Delta B_{2, k}, \\
P_{k+1}=P_{k}+\left[D(r(i \Delta t))\left(P_{0}-P_{k}\right)-h_{3} X_{k} P_{k}\right] \Delta t+\sigma_{3}(r(i \Delta t)) P_{k} \Delta B_{3, k},
\end{array}\right.
$$



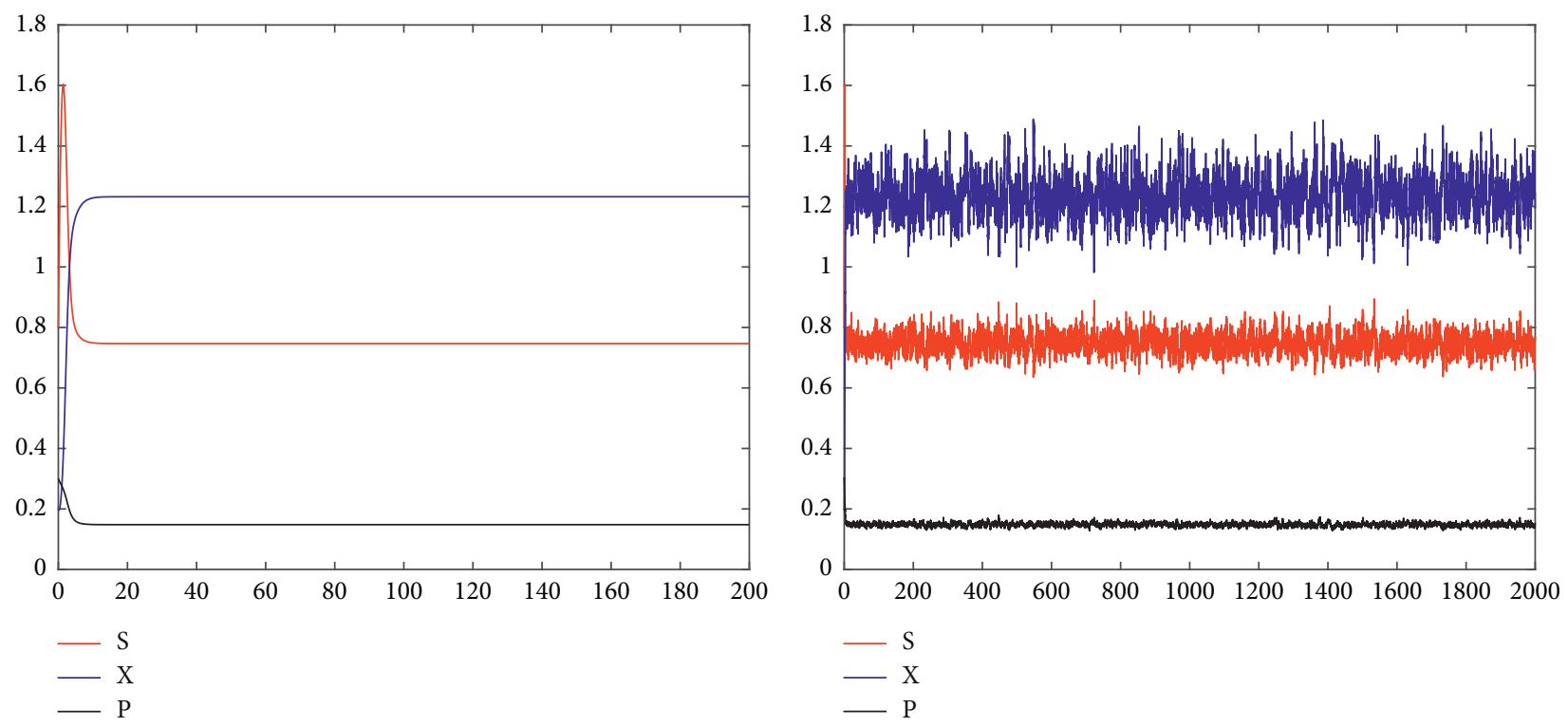

(a)

(b)

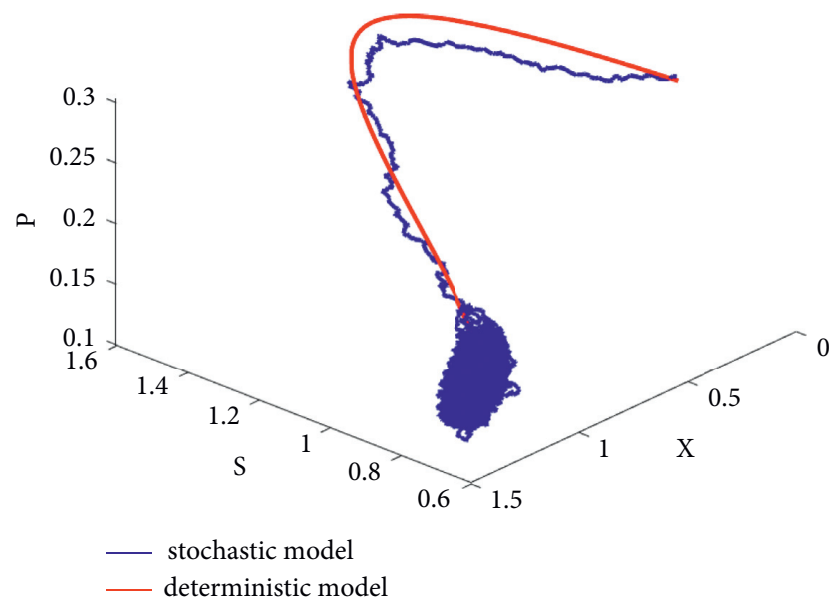

(c)

Figure 2: The comparison of solutions for system (1) and system (4). (a) The solutions of system (1) with $\tau=0$, (b) the solutions of system (4), and (c) the comparison of phase portrait for system (1) and system (4).

where $\Delta B_{i, k} \triangleq B\left(t_{k+1}\right)-B\left(t_{k}\right)(i=1,2,3)$ obeys the Gaussian distribution $N(0, \Delta t)$.

Firstly, we consider the cases of two regimes, without loss of generality, we let $k=1$ and $k=2$, and the switching between them is governed by $r(t)$ in the state space $\mathbb{S}=$ $(1,2)$ with the generator

$$
\left(\begin{array}{cc}
-6 & 6 \\
4 & -4
\end{array}\right)
$$

A direct computation shows the stationary distribution is $\pi=\left(\pi_{1}, \pi_{2}\right)=(0.4,0.6)$.

Example 1. For system (3), the parameters are chosen as

$$
\begin{aligned}
S_{0} & =3.2, \\
P_{0} & =1.2, \\
\mu_{1} & =1.6, \\
\mu_{2} & =1.2, \\
h_{2} & =2, \\
h_{3} & =0.5, \\
D(1) & =0.5, \\
\sigma_{1}(1) & =\sigma_{2}(1)=\sigma_{3}(1)=0.05, \\
D(2) & =0.6, \\
\sigma_{1}(2) & =\sigma_{2}(2)=\sigma_{3}(2)=0.05,
\end{aligned}
$$

with the initial values $(S(0), X(0), P(0))=(0.8,0.2,0.3)$. 

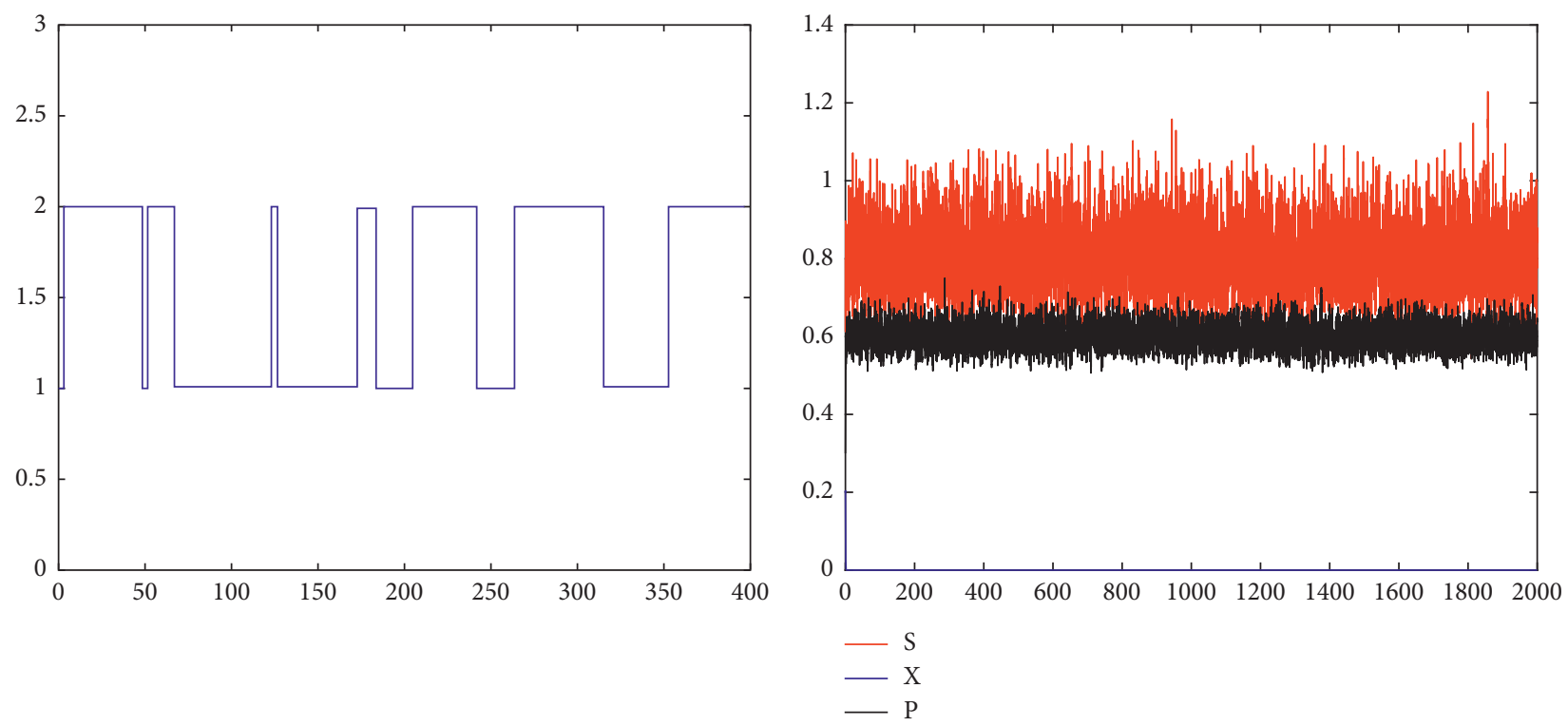

(a)

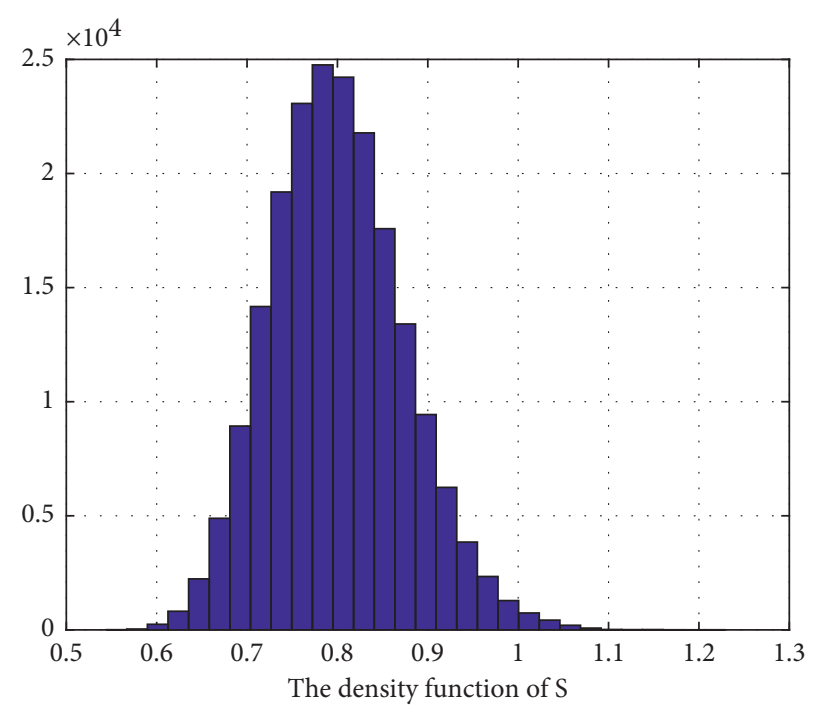

(c) (b)

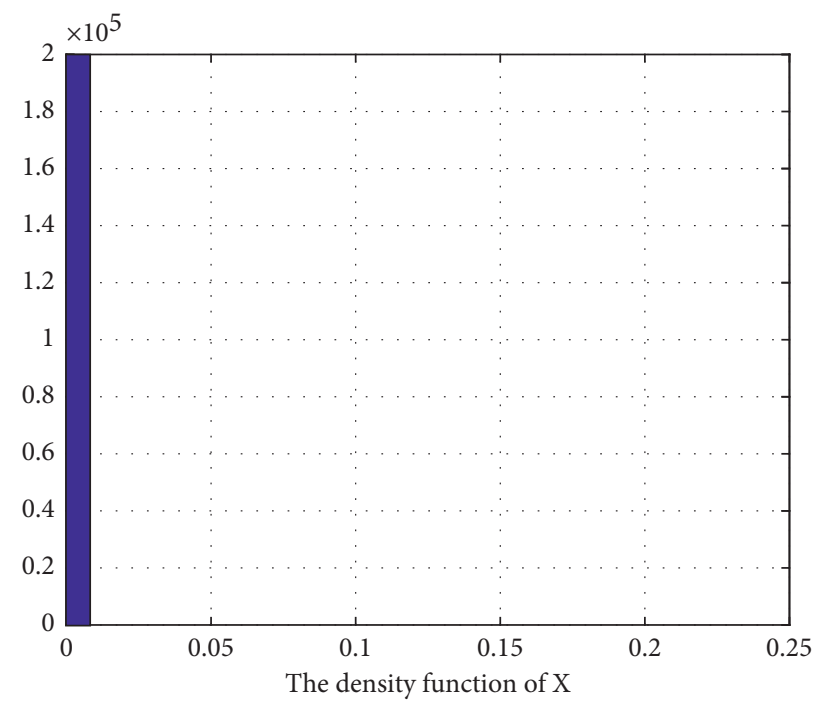

(d)

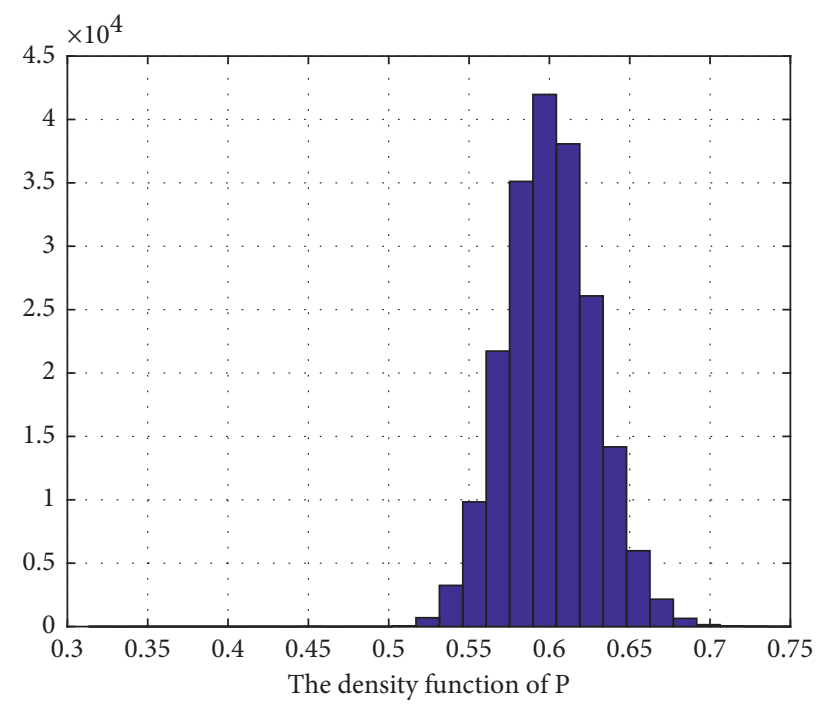

(e)

Figure 3: The numerical simulation of solutions of system (4). (a) Markov chain, (b) the solution of system (4), (c) the density function of $S(t)$ in system (4), (d) the density function of $X(t)$ in system (4), and (e) the density function of $P(t)$ in system (4). 
A direct computation shows $\Lambda=\sum_{k \in \mathbb{S}} \pi_{k} \Lambda \quad(k)$ $=0.8702>0$, and then, by Theorem 2 , system (4) has a unique ergodic stationary distribution (see Figure 1). Compared the solutions of the deterministic system, it can be seen that the stochastic disturbance has a significant impact on system (4) (see Figure 2).

Example 2. For system (4), choose the parameters as

$$
\begin{aligned}
S_{0} & =0.8, \\
P_{0} & =0.6, \\
\mu_{1} & =1.8, \\
\mu_{2} & =1.4, \\
h_{2} & =2, \\
h_{3} & =0.5, \\
D(1) & =2, \\
\sigma_{1}(1) & =0.15, \\
\sigma_{2}(1) & =0.2, \\
\sigma_{3}(1) & =0.1, \\
D(2) & =2.4, \\
\sigma_{1}(2) & =0.2, \\
\sigma_{2}(2) & =0.24, \\
\sigma_{3}(2) & =0.1,
\end{aligned}
$$

and the initial values are same as Example 1.

By simple computation, we get $\mu_{2} S_{0}(\check{D} / \widehat{D})+P_{0}(\check{D} / \widehat{D})=$ $2.064<\sum_{k \in \mathbb{S}} \pi_{k}\left[D(k)+(1 / 2) \sigma_{2}^{2}(k)\right]=2.26528$, and according to Theorem 3 , the microorganism will eventually become extinct (see Figure 3).

\section{Conclusion}

In this paper, focusing on the dynamics of the microbial flocculation process, a new type of chemostat model with flocculation effect is proposed from a random perspective. The stochastic dynamic properties of the system are investigated under regime switching conditions. The existence and ergodicity for a stationary distribution of the system and the extinction of the microorganisms are proved, and the corresponding sufficient conditions are obtained. Moreover, the theoretical results are illustrated by computer simulation. Our results enrich the research work of microbial culture and flocculation problems. However, due to the complexity of the model, the durability of the system has not been proven, and we leave this issue for future research.

\section{Data Availability}

No data were used to support this study.

\section{Conflicts of Interest}

The authors declare that there are no conflicts of interest regarding the publication of this study.

\section{Acknowledgments}

This work was supported by Scientific Research Foundation of Shandong University of Science and Technology for Recruited Talents.

\section{References}

[1] G. R. Rose and M. R. S. John, "Flocculation," Encyclopedia of Polymer Science and Technology, 2011, https://onlinelibrary. wiley.com/doi/abs/10.1002/0471440264.pst136.

[2] H.-J. Choi, "Effect of mg-sericite flocculant for treatment of brewery wastewater," Applied Clay Science, vol. 115, pp. 145-149, 2015.

[3] D. V. Dixon, S. R. Stoyanov, Y. Xu, H. Zeng, and J. B. P. Soares, "Challenges in developing polymer flocculants to improve bitumen quality in non-aqueous extraction processes: an experimental study," Petroleum Science, vol. 17, no. 3, pp. 811-821, 2020.

[4] J. Qiu, W. Zheng, B. Wu, and J. Wang, "The application of microbial flocculant in aquaculture wastewater treatment," Journal of Biology, vol. 28, no. 6, pp. 98-101, 2011.

[5] B. S. Gupta and J. E. Ako, "Application of guar gum as a flocculant aid in food processing and potable water treatment," European Food Research and Technology, vol. 221, no. 6, pp. 746-751, 2005.

[6] Z.-B. Wu, W.-M. Ni, and B.-H. Guan, "Application of chitosan as flocculant for coprecipitation of $\mathrm{mn}$ (ii) and suspended solids from dual-alkali fgd regenerating process," Journal of Hazardous Materials, vol. 152, no. 2, pp. 757-764, 2008.

[7] D. M and B. S, "Synthesis, characterization and application of acryloyl chitosan anchored copolymer towards algae flocculation," Carbohydrate Polymers, vol. 152, pp. 459-467, 2016.

[8] R. Fekih-Salem, J. Harmand, C. Lobry, A. Rapaport, and T. Sari, "Extensions of the chemostat model with flocculation," Journal of Mathematical Analysis and Applications, vol. 397, no. 1, pp. 292-306, 2013.

[9] X. Tai, W. Ma, S. Guo, H. Yan, and C. Yin, "A class of dynamic delayed model describing flocculation of micoorganism and its theoretical analysis," Mathematics in Practice and Theory, vol. 45, no. 13, pp. 198-209, 2015, in Chinese.

[10] R. Fekih-Salem, A. Rapaport, and T. Sari, "Emergence of coexistence and limit cycles in the chemostat model with flocculation for a general class of functional responses," $A p$ plied Mathematical Modelling, vol. 40, no. 17, pp. 7656-7677, 2016.

[11] S. Guo, W. Ma, and W. Ma, "Global dynamics of a microorganism flocculation model with time delay," Communications on Pure and Applied Analysis, vol. 16, no. 5, pp. 1883-1891, 2017.

[12] S. Guo, W. Ma, and X.-Q. Zhao, "Global dynamics of a timedelayed microorganism flocculation model with saturated functional responses," Journal of Dynamics and Differential Equations, vol. 30, no. 3, pp. 1247-1271, 2018.

[13] K. Song, T. Zhang, and W. Ma, "Nontrivial periodic solution of a stochastic non-autonomous model with biodegradation of microcystins," Applied Mathematics Letters, vol. 94, pp. 87-93, 2019.

[14] H. Zhang and T. Zhang, "The stationary distribution of a microorganism flocculation model with stochastic perturbation," Applied Mathematics Letters, vol. 103, Article ID 106217, 2020. 
[15] H. Zhang and T. Zhang, "Asymptotic behavior of a stochastic microorganism flocculation model with time delay," Applied Mathematics Letters, vol. 121, Article ID 107384, 2021.

[16] S. Guo, J.-A. Cui, and W. Ma, "An analysis approach to permanence of a delay differential equations model of microorganism flocculation," Discrete \& Continuous Dynamical Systems - B, 2021, https://www.aimsciences.org/article/doi/10. 3934/dcdsb.2021208.

[17] R. Liu and W. Ma, "Noise-induced stochastic transition: a stochastic chemostat model with two complementary nutrients and flocculation effect," Chaos, Solitons \& Fractals, vol. 147, Article ID 110951, 2021.

[18] R. Yan and S. Sun, "Stochastic characteristics and optimal control for a stochastic chemostat model with variable yield," Complexity, vol. 2020, Article ID 5065172, 18 pages, 2020.

[19] C. Xu, S. Yuan, and T. Zhang, "Competitive exclusion in a general multi-species chemostat model with stochastic perturbations," Bulletin of Mathematical Biology, vol. 83, pp. 1-17, 2021.

[20] T. Zhang, N. Gao, J. Wang, and Z. Jiang, "Dynamics system of microbial culture described by impulsive differential equations," Mathematical Modeling and Its Applications, vol. 8, no. 1 , p. 52, 2019, in Chinese.

[21] C. Xu, S. Yuan, and T. Zhang, "Average break-even concentration in a simple chemostat model with telegraph noise," Nonlinear Analysis: Hybrid Systems, vol. 29, pp. 373-382, 2018.

[22] C. Ji, "The stationary distribution of hepatitis B virus with stochastic perturbation," Applied Mathematics Letters, vol. 100, Article ID 106017, 2020.

[23] Q. Liu and D. Jiang, "Stationary distribution and extinction of a stochastic predator-prey model with distributed delay," Applied Mathematics Letters, vol. 78, pp. 79-87, 2018.

[24] M. Gao and D. Jiang, "Ergodic stationary distribution of a stochastic chemostat model with regime switching," Physica A: Statistical Mechanics and Its Applications, vol. 524, pp. 491-502, 2019.

[25] Q. Liu, D. Jiang, and N. Shi, "Threshold behavior in a stochastic siqr epidemic model with standard incidence and regime switching," Applied Mathematics and Computation, vol. 316, pp. 310-325, 2018.

[26] X. Yu, S. Yuan, and T. Zhang, "Asymptotic properties of stochastic nutrient-plankton food chain models with nutrient recycling," Nonlinear Analysis: Hybrid Systems, vol. 34, pp. 209-225, 2019.

[27] L. Wang and D. Jiang, "Ergodic property of the chemostat: a stochastic model under regime switching and with general response function," Nonlinear Analysis: Hybrid Systems, vol. 27, pp. 341-352, 2018.

[28] H. Liu, X. Li, and Q. Yang, "The ergodic property and positive recurrence of a multi-group Lotka-Volterra mutualistic system with regime switching," Systems \& Control Letters, vol. 62, no. 10 , pp. 805-810, 2013.

[29] R. Z. Khasminskii, C. Zhu, and G. Yin, "Stability of regimeswitching diffusions," Stochastic Processes and Their Applications, vol. 117, no. 8, pp. 1037-1051, 2007.

[30] R. S. Liptser, "A strong law of large numbers for local martingales," Stochastics, vol. 3, no. 1-4, pp. 217-228, 1980.

[31] Y. Zhao and D. Jiang, "The threshold of a stochastic sis epidemic model with vaccination," Applied Mathematics and Computation, vol. 243, pp. 718-727, 2014. 